\title{
Eliminación de estadíos juveniles de Ascaris lumbricoides (Linneo, 1758) por vía oral. Reporte de un caso y algunas consideraciones epidemiológicas
}

\author{
Patricia Neira O., Gloria Pino Q., Nelson Muñoz S. y Pedro Tobar C.
}

\section{Elimination of juvenile stages of Ascaris lumbricoides (Linnaeus, 1758) by mouth. Report of a case and some epidemiological considerations}

Ascariosis is a parasitic disease caused by Ascaris lumbricoides, a large geohelmint endemic in our country. At present, ascariosis is a rare infection in Chile. We present a case of an adult, resident of the Villa Alemana municipality, Valparaíso Region, who spontaneously expelled two juvenile nematodes by mouth. We review the manifestations produced by the larval and adult stages of this parasite including their diagnosis, treatment, and epidemiological considerations.

Key words: Ascariosis, Ascaris lumbricoides, roundworm, diagnosis.

Palabras clave: Ascariosis, Ascaris lumbricoides, nemátodo, diagnóstico.

\section{Introducción}

\section{L} a ascariosis es una parasitosis producida por Ascaris lumbricoides, gusano cilindroídeo de extremos aguzados, de la familia Ascarididae, orden Ascaridida, clase Secernentea. Es un nemátodo que parasita exclusivamente al ser humano. El hombre lo adquiere al ingerir huevos en el segundo estadío juvenil desde el ambiente contaminado con excretas ${ }^{1}$.

La infección se denomina ascariosis o ascaridiasis. La mayoría de los casos se presenta en los niños y los adolescentes, lo que sugiere una mayor exposición al ambiente contaminado y una aún deficiente higiene personal en la ingesta alimentaria. En adultos, en cambio, es poco frecuente ${ }^{2}$.

Presentamos el caso clínico de una mujer en período de lactancia, que eliminó por vía oral dos ejemplares en estadíos juveniles de $A$. lumbricoides, en su fase posterior al ciclo de Loos y se discute las circunstancias más relevantes.

\section{Caso clínico}

Mujer de 29 años de edad, sana, sin antecedentes mórbidos de importancia, en proceso de amamantamiento de su hijo de cuatro meses. Procedente de la zona suburbana de Villa Alemana, Región de Valparaíso. Vive en una casa que dispone de agua potable y alcantarillado; posee un perro, dos gatos y un conejo como mascotas. Como dueña de casa, es ella quien prepara los alimentos. En el último mes, separado por quince días, había efectuado dos viajes a la ciudad de Talcahuano, Concepción, a consecuencias del desastre ocurrido en la casa de un familiar por el terremoto y tsunami de febrero de 2010.

La paciente acudió a Consultorio Periférico de Villa Alemana con dos ejemplares de gusanos eliminados por la boca hacía siete días (Figura 1a). Relató que se encontraba durmiendo y despertó al sentir una sensación muy extraña en su garganta y luego en su boca; frente al movimiento de un cuerpo extraño en la boca, con su lengua lo aprisionó contra el paladar para retirarlo con sus dedos. Lo dejó sobre su velador donde observó que se enroscaba. Posteriormente eliminó un segundo ejemplar; en ambos casos sin tos, expectoración ni vómito. Durante el día había presentado malestar general, náuseas, diarrea acuosa, hiporexia, dispepsia, meteorismo y fiebre. Relató haber consumido merluza cruda (cebiche) once días atrás y negó el consumo de verduras crudas en la familia. La familia integrada por seis componentes: su esposo, su hermana (18 años), tres hijos de nueve y cinco años y el menor de cuatro meses, no presentaron signos ni síntomas. El médico tratante le indicó terapia con mebendazol 100 mg, dos veces al día, por tres días.

En el laboratorio del consultorio, por las características morfométricas se sospechó que los ejemplares correspondían a A. lumbricoides en estado juvenil. Por igual motivo se efectuó examen copro-parasitario seriado, que resultó negativo.

Uno de los ejemplares de los gusanos eliminados fue derivado al laboratorio de Parasitología de la Escuela de Medicina de la Universidad de Valparaíso para comprobar el diagnóstico. Se realizó el estudio morfométrico,
Universidad de Valparaíso, Chile.

Facultad de Medicina,

Escuela de Medicina, Departamento de Preclínicas. Cátedra de Parasitología (PNO, GPQ, NMS) Laboratorio Clínico Consultorio de Villa Alemana, Región de Valparaíso, Chile (PTC).

Fuente de Financiamiento: Fondos institucionales.

Los autores declaran que no existe conflicto de interés.

Recibido: 13 de septiembre de 2010 Aceptado: 1 de agosto de 2011

Correspondencia a:

Patricia Neira O.

patricia.neira@uv.cl 


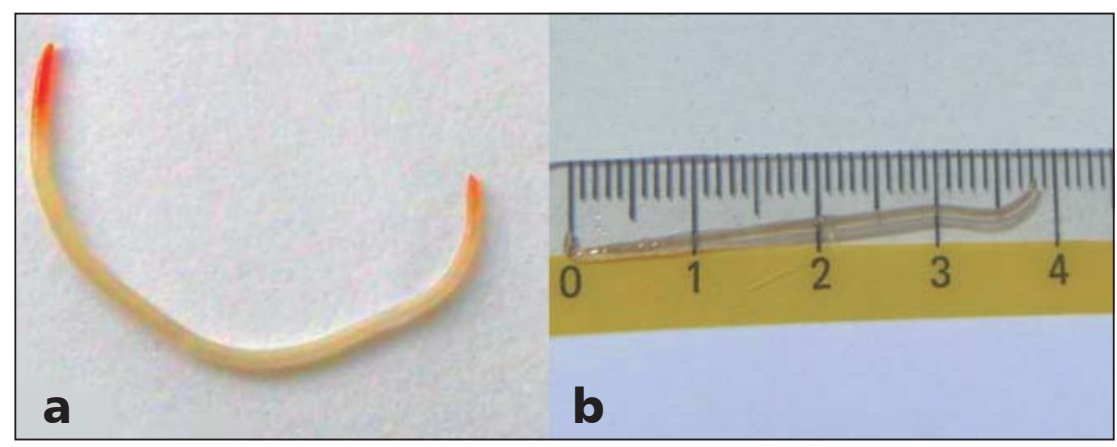

Figura 1. Ejemplar juvenil de Ascaris lumbricoides eliminado por vía oral (a) y su longitud en cm (b).

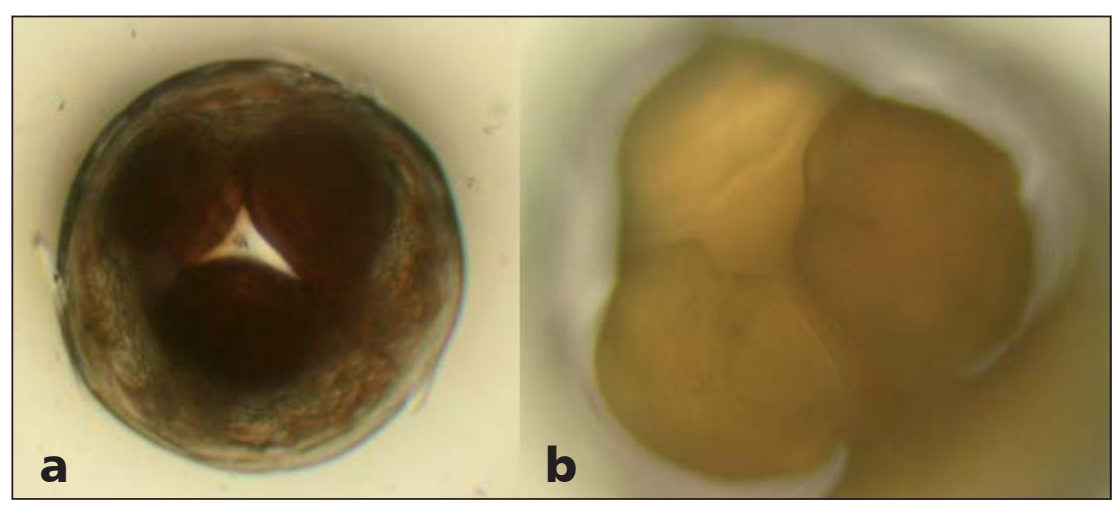

Figura 2. a. Visión frontal de extremo anterior de Ascaris lumbricoides, mostrando los tres labios y cavidad oral triangular, b. diafanizado con lacto-fenol de Aman (Aumento 4x).

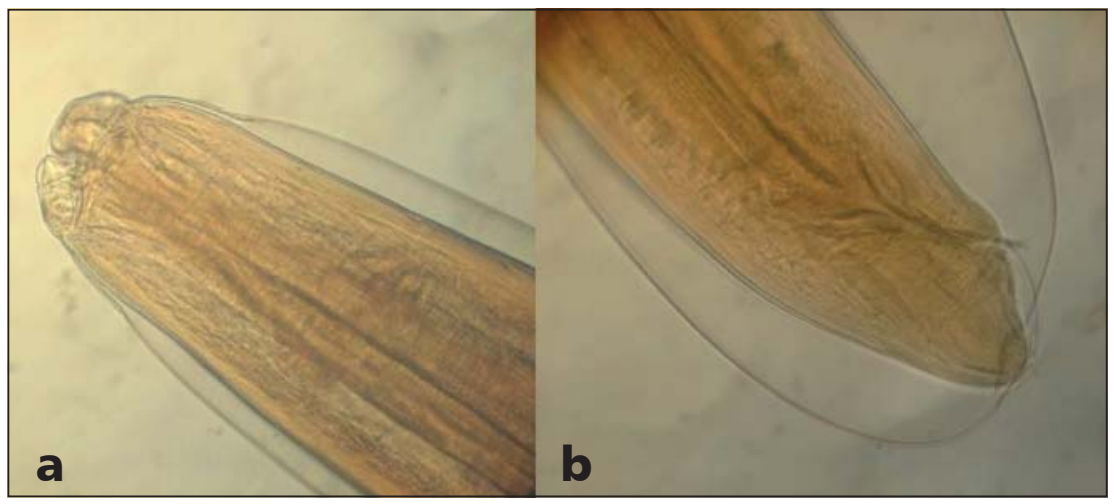

Figura 3. Extremo anterior (a) y posterior (b) de Ascaris lumbricoides juvenil (Aumento 3,5x).

destacando su color amarillento, una forma cilíndrica de extremos aguzados rojizos, de $3,9 \mathrm{~cm}$ de longitud y 1,0 mm de ancho (Figura 1a y b). Microscópicamente, se comprobó la presencia de tres labios en el extremo anterior; uno dorsal con dos papilas y con sólo una cada uno en los dos labios ventrolaterales ${ }^{3}$ rodeando la cavidad oral (Figura 2 a y b).

Con el objeto de una mejor observación de las características del gusano, el ejemplar fue sometido a diafanización en lacto-fenol de Amann. El diagnóstico fue corroborado al visualizar microscópicamente los tres labios que rodean la cavidad oral triangular en el extremo anterior (Figura 2), y por las características del tubo digestivo por transparencia (esófago corto, intestino aplanado recorriendo toda la longitud del cuerpo, terminado en recto) (Figura 3).

Obtenido el diagnóstico de certeza, se sostuvo entrevista telefónica con la paciente: se le indicó las medidas profilácticas para evitar la reinfección y se le sugirió control mediante examen copro-parasitario seriado, el que reveló formas vacuoladas de Blastocystis hominis. Como investigación complementaria se efectuó examen copro-parasitario seriado a los integrantes de su grupo familiar, evidenciándose formas vacuoladas de B. hominis y quistes de Entamoeba coli en su hermana; en el resto de los integrantes el estudio fue negativo.

\section{Discusión}

Ascaris lumbricoides, comúnmente denominado lombriz, es un nemátodo polimiario cuyo hábitat es el intestino delgado del ser humano, donde se mantiene nadando contra la corriente. Conocido desde los comienzos de la civilización, los huevos han sido encontrados en coprolitos humanos de Perú que datan desde 2277 A. de C. ${ }^{4}$. Los egipcios hacen referencia a este geohelminto en el papiro de Ebers. Los griegos los llamaron gusano redondo, en inglés "roundworm", lo que no corresponde puesto que, morfológicamente presentan forma cilíndrica con extremos aguzados; sin embargo, la sección transversal es circular. Por la similitud que detectaron los autores romanos con la lombriz común de tierra Lombricus terrestres (anélido, oligoqueto, de vida libre), se referían a ellos como Lombricus teres o gusanos redondos, para diferenciarlos de las lombrices aplanadas Lombricus latus 5 .

Se caracteriza por tener un cuerpo blando, de color blanco, a veces amarillento o rojizo, según las sustancias ingeridas por el hospedador. Presenta dimorfismo sexual, las hembras son de mayor tamaño que los machos y en el macho, el extremo posterior se encuentra enroscado ventralmente.

Su ciclo es bien conocido, presenta distribución mundial especialmente en zonas tropicales y subtropicales; es prevalente y endémico en áreas desprovistas o con infraestructura sanitaria deficiente.

En Chile, la parasitosis se presenta habitualmente en forma asintomática y cuando existe un cuadro clínico presenta características totalmente inespecíficas. Dependiendo de la cantidad de gusanos presentes y de eventuales complicaciones que pudieran presentarse, podríamos asistir a la aparición de signos y síntomas vagos. En la 
fase larvaria o pulmonar: tos espasmódica, expectoración mucosa, fiebre, hemoptisis ocasional y respiración sibilante; en la fase adulta o intestinal: vómitos, náuseas, pérdida de apetito, diarrea, distensión abdominal, dolor abdominal tipo cólico localizado en epigastrio, fiebre y cuadros alérgicos.

La migración de áscaris hacia la parte alta del tubo digestivo se produce en algunas oportunidades en forma espontánea y en otras, porque el ambiente se torna desfavorable, como es el caso de fiebre, ingesta de alcohol, náuseas, y vómitos, inhalación de anestésicos (éter, cloroformo), la presencia de algunos fármacos (vermífugos) o determinados alimentos, que provocan la excitación del parásito. La eliminación de áscaris por la nariz durante la anestesia con éter en niños y en el vómito de algunos enfermos en el post operatorio precoz, fue mencionada por De la Fuente en $1957^{6}$.

El parásito puede efectuar una migración que podría finalizar con la expulsión del gusano al exterior a través del ano, la boca o la nariz. Durante la migración junto con la oclusión de los conductos, el áscaris puede acarrear consigo bacterias intestinales capaces de producir abscesos de los órganos obstruídos ${ }^{7}$. Otras complicaciones son: pseudo-obstrucción intestinal (ascariosis masiva) ${ }^{8}$, obstrucción de las vías biliares (que incluyen el hígado y la vesícula biliar) o pancreática, muy poco frecuentes en Chile ${ }^{2,9}$.

La ascariosis pulmonar puede ser diagnosticada por el hallazgo del estadío juvenil en el esputo varios días post infección, como fuera demostrado por Koino en 1922, al infectar a voluntarios y auto-infectarse con 2.000 huevos del nemátodo; 50 días después recuperó de su intestino 667 gusanos inmaduros por administración de un antihelmíntico ${ }^{10}$. El estado juvenil también puede diagnosticarse en el aspirado gástrico durante la fase migratoria ${ }^{11}$.

En una revisión de la literatura médica, no encontramos referencias de eliminación de A. lumbricoides juvenil por vía oral, (es posible que ello se deba a que, siendo la eliminación de gusanos por boca o ano relativamente frecuentes no fue considerado de importancia como para su publicación); sólo se hace mención de esta condición en los libros de texto. Existe un reporte de la eliminación por la boca de un ejemplar adulto en un ciudadano español ${ }^{12}$.

Los signos y síntomas que presentó la paciente y las características morfométricas del ejemplar analizado, nos permiten inferir que los estadíos juveniles eliminados por la boca, procedían del tubo digestivo en su fase posterior al ciclo de Loos.

Diagnóstico: El diagnóstico de certeza de esta geohelmintiasis se logra por la observación de los estadíos adultos eliminados principalmente por el ano y excepcionalmente por la boca ${ }^{12}$; los estadíos juveniles eliminados por la boca, como sucedió en el caso relatado o por la observación de los huevos fértiles o infértiles (si existen machos y hembras, o sólo si están presentes las hembras en el intestino, respectivamente) en las deposiciones a través de un examen copro-parasitario seriado. El estudio de las deposiciones será negativo para la presencia de huevos: si sólo existen machos en el intestino, si los estadíos juveniles intestinales no han alcanzado la madurez o si éstos se encuentran en el ciclo de Loos (ciclo extra-intestinal).

La mayor dificultad para el diagnóstico de certeza se presenta cuando el parásito es eliminado en un estadío juvenil, como sucedió en este caso, ya que se podría sospechar de otras helmintiasis como anisakiosis, entre otras.

El diagnóstico diferencial debe efectuarse con estadíos juveniles de helmintos de la Familia Anisakidae, que pertenecen también al orden Ascaridida, y que son eliminados por la boca ${ }^{13}$, escasos días después de haber ingerido pescado crudo o insuficientemente cocido (esta paciente había ingerido merluza cruda, como cebiche, 11 días atrás).

El tratamiento debe incluir medidas de soporte (sintomáticas) y fármacos anti-helmínticos, siendo de elección el mebendazol (dosis de $100 \mathrm{mg}$ dos veces al día durante tres días) o albendazol (una sola dosis de 400 mg). Además métodos preventivos para evitar la reinfección y un examen copro-parasitario seriado de control al mes post tratamiento.

Consideraciones epidemiológicas: En 2008, la OMS estimó en 980 millones las personas parasitadas por $A$. lumbricoides ${ }^{14}$. La distribución geográfica de la helmintiasis es variable según los niveles de vida de la población, predominando en países tropicales con condiciones de vida y saneamiento deficientes. La prevalencia en pueblos aborígenes de Malasia fluctúa entre 4,6 a 86,7\% ${ }^{15}$; en Brasil diversos estudios demuestran prevalencias de 5,8 a 59,5\% ${ }^{16-17}$. En Etiopía un estudio reciente demostró 22\% de ascaridiasis ${ }^{18}$. Por otra parte, la frecuencia aumenta frente a situaciones de desastres naturales ${ }^{19}$.

Hace poco más de 50 años, una de las infecciones más frecuentes que debían afrontar los profesionales de la salud de nuestro país, era la ascariosis, especialmente en los infantes y adolescentes. La prevalencia de A. lumbricoides, presentaba un ascenso progresivo de norte a sur del país, con diferente magnitud entre las zonas urbanas y las rurales, explicable en parte por la influencia del ambiente natural (factores climáticos como la sombra, la temperatura y la humedad), la calidad físico-química del suelo, la hidrografía y topografía regional, el tipo de vegetación y la altura sobre el nivel del mar y, por otra parte, la contaminación fecal del suelo y los hábitos higiénicos deficientes de la población ${ }^{20}$. En la zona norte (regiones de Tarapacá y Atacama), la prevalencia fluctuaba entre 0,2 y 2,4\%; de Coquimbo al Maule oscilaba entre 23,3 y 
46,4\% y en el sur (provincias de Arauco y Chiloé) entre 50 y $60 \%$ en algunos sectores ${ }^{21-23}$.

En los años siguientes, estos helmintos que se transmiten a través del suelo, mostraron una clara tendencia decreciente de su prevalencia, lo que se asoció, por una parte, a una evidente mejoría en las condiciones de saneamiento ambiental (suministro de agua potable y disposición de excretas), tanto en los sectores urbanos como rurales, acaecidas en el país en el periodo 19701980 y por otra, a la mejora de las condiciones generales de vida y de higiene, la disminución del analfabetismo, al uso de nuevos fármacos anti-helmínticos, así como a la redistribución de la población por la migración hacia las ciudades ${ }^{24,25}$.

En la actualidad, aunque en algunas zonas persisten deficiencias en el saneamiento ambiental, se aprecia un evidente progreso en la edificación y la urbanización; esto último ha modificado notablemente la composición físicoquímica del suelo. Estos avances, sumados a la mejoría en los sistemas de disposición de excretas, han restringido en forma paulatina las áreas de tierras húmedas y en muchos sectores se ha reducido la contaminación fecal humana del suelo, lo que ha permitido la disminución progresiva de algunas enteroparasitosis, entre las que se encuentra la ascariosis.

La reciente comunicación de Vidal y cols, muestra un marcado descenso de 10,3\% en el quinquenio 1980-1984 a 0,11\% en los años 2005-2008 de A. lumbricoides en la ciudad de Talca ${ }^{26}$.

En la Región de Valparaíso, en 1987 se comunicaba la ausencia de A. lumbricoides en escolares de Algarrobo, Casablanca y Colliguay ${ }^{27-29}$, a pesar de que las condiciones ambientales de la zona favorecían su desarrollo. En el Ar- chipiélago de Juan Fernández las condiciones ambientales no son iguales a las continentales, pero presentan similares enteroparasitosis al continente, sin embargo; los estudios han mostrado ausencia de $A$. lumbricoides ${ }^{30,31}$.

\section{Conclusiones}

Ascaris lumbricoides es una geo-helmintiasis que actualmente presenta baja frecuencia en Chile. Se asocia a deficiente saneamiento básico y la consecuente contaminación fecal del suelo pero también a la cultura higiénica de las personas, la que pareciera tener más importancia en la variación de su frecuencia en la población. La prevalencia es mayor en los niños y los adolescentes, los adultos rara vez son afectados. El diagnóstico se certifica por la observación de huevos en las deposiciones y/o los estadíos juveniles o adultos eliminados por boca o ano. El tratamiento se realiza con mebendazol.

\section{Resumen}

La ascariosis es una parasitosis provocada por Ascaris lumbricoides, el geo-helminto de mayor tamaño que afecta al ser humano en nuestro país. En Chile, la ascariosis es una infección poco frecuente en la actualidad. Se presenta el caso de un adulto residente en la comuna de Villa Alemana, Región de Valparaíso, que eliminó en forma espontánea dos ejemplares del nemátodo en estado juvenil por vía oral. Se revisan las manifestaciones producidas por este parásito en la fase larvaria y adulta, el diagnóstico, el tratamiento y algunas consideraciones epidemiológicas.

\section{Referencias}

1.- Subercaseaux B, Neira P. Ascaridiasis en: Subercaseaux B, Neira P. Apuntes de Parasitología Práctica. Universidad de Valparaíso Editorial. 1993: 92-5.

2.- Morales G J L, Arpon G F, Bravo C C. Obstrucción intestinal por Ascaris lumbricoides. Rev Chil Cir 2009; 61: 13-4.

3.- Guiart J. Nematoides intestinales: Ascaridiosis. En Guiart J. Manual de Parasitología. Barcelona España. Salvat y C. a en C. Editores; 1915, p. 384-403.

4.- Horne P D. A review of the evidence for human endoparasitism in the pre-Columbian New World through the study of coprolites. J Archaeol Sci 1985; 12: 299-310.

5.- $\quad$ Grove D. Chapter 19: Ascaris lumbricoides y ascariasis. En Grove D. A history of human helminthology. United Kingdom, C.A.B. InternationalWallingford; 1990, p. 469-97.
6.- De la Fuente H. Ascaridiasis de las vías biliares. Bol Chil Parasitol 1957; 12: 32-4.

7.- Vera M, Vera W. Ascaridiasis quirúrgica con especial referencia a los cuadros de abdomen agudo. Pediatría 1959; 2:22-33.

8.- Vásquez Tsuji O, Gutiérrez Castrellón P, Yamazaki Nakashimada M A, Arredondo Suárez J C, Campos Riveral T, Martínez Barbosa I. Antihelmínticos como factor de riesgo en la obstrucción intestinal por Ascaris lumbricoides en niños. Bol Chil Parasitol 2000; 55: 3-7.

9.- Verdugo R, Mauret E. Invaginación intestinal por Ascaris lumbricoides presentación de un caso clínico. Rev Ped Elec 2009; 6: 180.

10.- Koino S. Experimental infections on the human body with ascarides. Japan Medical World 1922; 15: 317-20.

11.- Proffit R D, Walton B C. Ascaris pneumonia in a two-year-old girl. Diagnosis by gastric aspirate. N Engl J Med 1962; 266: 931-4.

12.- Nuño Mateo F J, Noval Menéndez J, Fonseca
Aizpuru E M, Otero Guerra, L. Varón de 25 años de edad con vómito con gusano e hinchazón facial. Rev Clin Esp 2003; 203: 305-6.

13.- Jofré M L, Neira O P, Noemí H I, Cerva C J L. Pseudoterranovosis y sushi. Rev Chil Infectol 2008; 25: 200-5.

14.- Melo M C B, Klem V G Q, Mota J A C, Penna F J. Parasitoses intestinais. Rev Med Minas Gerais 2004; 14 (Supl 1): S3-S12.

15.- Ahmed A, Al-Mekhlafi HM, Surin J. Epidemiology of soil-transmitted helminthiases in Malaysia. SoutheastAsian. J Trop Med Public Health 2011; 42: 527-38.

16.- Campos de Andrade E, Gonçalves I C, de Oliveira V, Goldner M. Parasitoses intestinais: um a revisão sobre seus aspectos sociais, epidemiológicos, clínicos e terapêuticos. Rev APS, Juiz de Fora 2010; 13: 231-40.

17.- Silva J C, Viana LF, Carvalho T, de Carvalho K, 
Pereira E, Fonseca A C. Parasitismo por Ascaris lumbricoides e seus aspectos epidemiológicos em crianças do Estado do Maranhão. Ver Soc Bras Med Trop 2011; 44: 100-2.

18.- Alemu A, Atnafu A, Addis Z, Shiferaw Y, Teklu T, Mathewos B, et al. Soil transmitted helminths and Schistosoma mansoni infections among school children in Zarima town, northwest Ethiopia. BMC Infect Dis 2011; 11 (1): 189.

19.- García Martínez E. Prevención y mitigación de desastres ante inundaciones en establecimientos de salud. OPS/USA/OFDA/Agencia Canadiense para el Desarrollo Internacional/ECHO s.l, Julio 2001; 120 págs. Disponible en http://www.crid. or.cr/digitalizacion/pdf/spa/doc14994/doc14994. htm (accedido en julio de 2011).

20.- Silva R. Alcances ecológicos en la epidemiología de las enfermedades parasitarias. Bol Chil Parasitol 1965; 20:113-22.

21.- Silva R, Donoso F, Neghme A. Consideraciones epidemiológicas sobre Ascaris lumbricoides en
Chile. I. Estudio en la región lacustre de Chile. Bol Chil Parasitol 1954; 9: 6-10.

22.- Neghme A, Silva R, Donoso F. Consideraciones epidemiológicas sobre Ascaris lumbricoides en Chile. II. La ascaridiasis en la zona norte del país. Bol Chil Parasitol 1954; 9: 47-50.

23.- Neghme A, Silva R. Distribución y frecuencia de las enteroparasitosis en Chile. Rev Serv Nac de Salud 1956; 1: 3-24.

24.- Ramírez R, Schenone H, Galdames M, Romero E, Inzunza E, Rojas A, et al. Frecuencia en Chile de las infecciones humanas por protozoos y helmintos intestinales (1962-1972). Bol Chil Parasitol 1972; 27: 116-8.

25.- Schenone H, Rojas A, Galdames M, Villarroel F. Aspectos epidemiológicos de las infecciones humanas por protozoos y helmintos intestinales en Chile (1970-1980). Bol Chil Parasitol 1981; 36:44-8.

26.- Vidal S, Toloza L, Cancino B. Evolución de la prevalencia de enteroparasitosis en la ciudad de Talca, Región del Maule, Chile. Rev Chil
Infectol 2010; 27: 336-40.

27.- Neira P, Villalón L, Muñoz N, Carabelli M, Tardío MT, Herrera G. Entero y ectoparasitosis en niños de la V Región, Chile. Estudio en el Internado “San José”, La Capilla, Algarrobo, 1986. Parasitol al Día 1987; 11:117-9.

28.- Neira P, Villalón L, Muñoz N, Herrera G. Incidencia de enteroparasitosis en población escolar de Casablanca V Región, 1986. Rev Med Valparaíso 1987; 40: 99-102.

29.- Tapia S, Muñoz N, Rojas J, Subercaseaux B. Estudio enteroparasitario en escolares del Valle de Colliguay. RevMed Valparaíso 1982; 35: 111-5.

30.- Astorga B, Briceño E, Jorquera H, Herskovic P. Perfil parasitológico en habitantes de la Isla Robinson Crusoe. Parasitol al Día 1988; 12: 176-81.

31.- Neira P, Muñoz N, Subercaseaux B, Tapia S, Herrera G. Enteroparasitosis en niños de la V Región. Estudio en el Archipiélago de Juan Fernández. Bol Chil Parasitol 1990; 45: 28-9. 